\title{
Logical Analogies
}

\author{
TRUDY COVIER University of Calgary
}

\begin{abstract}
"There is a natural and intuitive notion of recognizable form." Douglas Hofstadter, Godel, Escher, Bach, page 68.

"Some philosophers, such as Bertrand Russell, have believed that every assertion has just one essential logical form. They have held, for instance, that a sentence such as 'Brutus betrayed Caesar' cannot legitimately be regarded as having the logical form of an A sentence; they maintain that this kind of example is essentially relational in style..... But this attitude is misguided. It is only in the context of a specific argument that we can say that a sentence ought to be analyzed as, say, relational rather than categorical. In some other argument the same sentence might properly be analyzed in the opposite fashion." Stephen Barker in The Elements of Logic (Third Edition), page 74.
\end{abstract}

\begin{abstract}
"We defined the form of an argument as a set of arguments generable from it by variation, that is, by substitution for the elements designated as variable. An argument is valid if it satisfies the definition of validity, and invalid if the substitution yields any arguments whose premisses are true and conclusion false." Rolf George in "Bolzano's Consequence, Relevance, and Enthymemes," in Journal of Philosophical Logic, Vol. 12 (1983), page 320.
\end{abstract}

The technique of refuting arguments by constructing logically parallel ones seems to me to be interesting in a number of ways. For readers of this journal, chief among these may be the fact that is straddles the gap between formal and nonformal approaches to argument analysis. Like formal approaches, the technique is based on a perception that the argument refuted has a structure which is general. If that structure is shown flawed by the presentation of another argument which has the structure and is flawed, then the original argument is refuted. Like nonformal approaches, refutation by logical analogy does not require symbolization of the argument. Nor does it involve appeals to explicit rules of inference.

Interestingly, the logical analogy technique seems to be applicable to nondeductive arguments as well as to deductive ones. A theoretically perplexing question which the technique poses is whether an argument and its logical analogue do or do not share the same 'logical form'. Exploring this question reveals that preformal judgments are crucially involved when arguments are formalized: what we pick out as the correct logical form of an argument depends on insights into how that argument works - which are its significant and which its insignificant features. The very notion of form-and hence possibly also the basic contrast between formal and nonformal approaches to argument analysis-exhibits a puzzling indeterminacy.

First let us look at two examples of the technique of refutation by logical analogy. British doctor Penelope Leach, discussing the suitability of group child care for infants under three, argues as follows:

Many people would argue that while all of the foregoing is, or may be true, toddlers who are actually committed to group-care soon grow out of being 
toddlers and therefore become socialized more quickly than they would have done at home. 'The others will lick him into shape and he'll learn by imitating them...' People who take this line are usually those who very much want to believe that group care is acceptable for the very young, and who therefore use the observable fact that they do survive and develop, one way or another, as evidence to support it. So go back to that thirteen year-old who finds herself in charge of the family. [Leach alludes here to the case in which a mother dies and a girl of thirteen is charged with cooking for and caring for younger brothers and sisters.] She too will adapt. She too will learn 'how to behave', will find ways of managing and will, after a fashion, develop.

Does that prove such responsibilities are good for her? That these are the optimum conditions for adolescents and a useful way of short-circuiting its normally tumultuous path? No, of course not. Nobody would argue that, because nobody has any stake in thirteen-year-olds running families. But it is the same argument. Just as it is more appropriate for that girl to acquire maternal and household responsibilities out of mature sexuality rather than tragic deprivation, so it is better for the toddler to acquire socialized behaviour out of self-motivated maturity rather than sad necessity.[1]

Leach criticizes the argument from (1) children can adapt and develop normally when placed in group care very young to (2) group care is acceptable for the very young. She isolates the inference from (1) to (2) as the crux of an important argument defending group care for the very young. She then further isolates the 'essence' or 'core' of this inference when she constructs its logical analogy. In the logical analogy teen-age girls are substituted for toddlers and acquiring maturity from household responsibilities is substituted for socializing quickly and developing normally under group care. In the analogous argument, Leach insists, no one would be tempted to infer the conclusion from the premise. The logic- al parallel is without force; the argument about infant care is logically "the same argument"; therefore it too is without force. Such is the technique of refuting an argument by logical analogy.

My second example is taken from a philosophical source. In the following passage, Stanley Cavell uses a logical analogy to argue against C.L. Stevenson's defense of noncognitivism in ethics.

For then [that is, on a noncognitivist
account of ethics] we are going to have to
set up a display of humorous tolerance
and allow that some 'ethical' disagree-
ments cannot be 'settled' 'rationally'
on such grounds as this: whatever rea-
sons are offered them, when 'an over-
sexed, emotionally independent adoles-
cent argues with an undersexed, emo-
tionally dependent one about the desir-
ability of free love', their disagreement
may be 'permanently unresolved"'.
[Quotations are from Stevenson.] You
might as well say that if these two went
on permanently arguing about whether
men do or do not descend from apes,
then the science of biology would lack
an 'exhaustive' or 'definitive' method of
proof.
But it makes a difference whether the
argument in question is conducted by an
oversexed, emotionally dependent adult
like D.H. Lawrence, or an undersexed,
emotionally independent adult like
Freud-a difference which is part of the
nature of morality itself. It is a part of the
life of the subject that not every opinion
has the same weight nor every disagree-
ment the same significance.[2]

Cavell constructs his parallel to point out the extent to which Stevenson's argument is based on deriving permanent unresolvability from de facto failure (by two mis-matched individuals) to resolve. Stevenson then uses this conclusion to reach the further conclusion that the discipline in which the supposedly unresolvable dispute is located lacks a definite method of proof. Cavell substitutes biology for ethics in the argument, and varies the individuals, while preserving the poor 
match. He assumes that people will not find the logical analogy convincing, and since it is the same line of reasoning which appears in both arguments, they should not find the original argument convincing either.

Refutation by logical analogy is based on duplicating the 'core' of an argument in another argument by varying non-essential aspects while preserving essential ones. The parallel argument is exhibited to be, or argued to be, flawed. Seeing that it is flawed, we are to see the original argument as flawed also. The technique works best, obviously, when the logical analogue is clearly a parallel argument and is clearly flawed.

It is interesting to speculate whether there is any significant sense in which this technique may be said to expose the logical form of an argument. I suspect that there is, but that we would tend to resist this conclusion because we are so accustomed to associating logical form exclusively with structures built around standard logical terms such as "and," "or," "if then," "all," and so on.[3] (We are not accustomed to thinking of "can adapt," "is acceptable," "is not resolvable," and so on as logical words which might fix a structure common to several arguments.)

To explore this issue, I shall first depart from it by considering a very simple case in which two arguments quite uncontroversially share the same form.

The SAD Case:

SAD (a)

Joe is sad.

Peter is sad.

Therefore, Joe is sad and Peter is sad.

\section{SAD (b)}

Reagan is aggressive.

Thatcher is aggressive.

Therefore, Reagan is aggressive and Thatcher aggressive.
This SAD pair have a common form which can be represented as:

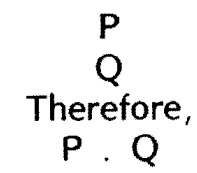

It is obvious that SAD (a) and SAD (b) share a common form. But why is this so 'obvious'? This is because the force of the argument in both SAD cases depends solely on the assertion of one statement, and then another, and then on the assertion in the conclusion of those two statements conjoined. There is just no other way to make these arguments 'flow' or 'hang together.' The particular individuals referred to have nothing to do with the force of the arguments; nor do the specific properties ascribed to them. This must be one of those easily recognizable forms which we can so naturally pick out-one of the ones Hofstadter had in mind when he said we have an "intuitive notion" of form. Our judgment that whether it is Peter or someone else who is sad in (a) does not matter-provided the individual appears in one premise and again in the conclusion-and that whether it is sadness or aggressiveness that is described does not matter, is a pre-formal judgment about the structure and logical direction of the argument. A formal representation of the argument as having the form ' $P$; $Q$; therefore $P . Q^{\prime}$ expresses this preformal judgment about what is significant and what is not significant in the nonformal statement of the argument.

This point is, perhaps, more apparent when we compare the SAD pair with another pair. 
The FAMOUS Case:

FAMOUS (a) FAMOUS (b)

Joe is famous. Sherlock Holmes is fictional.

Anyone who is famous is rich.

Therefore,

Joe is rich.

Anyone who is

fictional is real.

Therefore,

Sherlock Holmes is real

In this FAMOUS case, (a) is clearly a valid argument and may be formally represented as:

$$
\begin{gathered}
(\exists x) \text { (Jx.Fx) } \\
(x)(F x \rightarrow R x) \\
\text { therefore, } \\
(\exists x) \text { (Jx.Rx) }
\end{gathered}
$$

We would hesitate, however, to say that (b) shares this form. Even though (b) is, on one level, semantically parallel to (a), there is something very fishy about (b). We might judge that in (b) the second premise is clearly false, and see the difference between (a) and (b), and the unsoundness of (b), as due solely to this fact. We could, on such an account, regard (a) and (b) as sharing the above form. However, most philosophers will not wish to do this. They will hesitate because (b) uses "fictional" and "real" where (a) uses more standard predicates. To grant that (b) shares the same form as (a) will require ignoring this difference, and the difference seems too important to ignore in this way.

The FAMOUS case illustrates how pre-formal judgments can enter into decisions as to what the form of an argument is. Despite their surface similarity, FAMOUS (a) and FAMOUS (b) are not logically parallel arguments. In deductive logic-even elementary deductive logic-we rely on pre-formal judgments in order to formalize arguments and in order to determine whether two arguments share the same form. These judgments are about what is important and what is incidental in the arguments, so far as the move from premises to conclusion is concerned; and also about background issues such as that of whether "exists" and cognate terms are standard predicates. Formal analyses often help us to determine whether arguments are valid or invalid. However, they also serve to articulate preformal judgments of validity or invalidity and to defend these judgments. If the validity of an argument is pre-formally controversial, a treatment representing its form as one which is deductively valid will be controversial as well.

The technique of logical analogy relies on the same sense of the 'direction' of an argument as does formalization. We indicate our sense of what an argument depends on when we construct a parallel argument in which the central features of the original are preserved while its incidental features may be varied. In this case, we do not formalize in order to reveal the structure of the argument. Rather, we make structure appear by presenting a logical analogy. The structure or 'form' is repeated in the parallel argument. We 'see' it as we see sameness of shape in a blue circle and a red circle. The shape is common to both and can be seen as such without appearing as a separate structure.

In the light of the above considerations and an examination of other examples (appended below), I suggest the following reflections for your consideration:

1. The technique of logical analogy can in some cases provide a conclusive refutation of an argument. This fact merits further study and analysis.

2. The technique of logical analogy reveals that arguments depend on connections which are general. If this were not the case, the failure of the same connection to work in a parallel case would not show a flaw in the original argument.

3. The technique of logical analogy illustrates the fact that connections may 
be general without being, in the standard logician's sense, formal. The standard logician's sense of form relates to logical words such as "all," "some," "and," "or," "if then," and so on.

4. Because the thrust of such arguments as those criticized by Leach and Cavell does not crucially involve the workings of logical words as they are conventionally identified by logicians, the representation of such arguments in standard systems of deductive logic is relatively unhelpful.

5. The technique of logical analogy and that of formalizing simple arguments such as those in the SAD case depend on the same human skill: the recognition of the essential point of an argument, or what I have loosely termed its logical core. People are able to distinguish this core from content which may be varied while leaving the essence of the argument intact.

\section{ADDENDUM}

Further examples suggest that the technique of logical analogy is not restricted in its applicability to deductive arguments. Consider:

a. Here Arthur Pap is discussing Carnap's account of logical truth. On the basis of consideration of the example 'whatever is red is colored, he contends that Carnap's account is not applicable to natural languages. He then considers a possible rejoinder to his own argument and rejects the rejoinder by using a logical analagy:

\footnotetext{
I shall select a statement frequently used in discussions about the nature of a priori truth: 'whatever is red is colored'. The statement is, of course 'trivial', in the sense that it is obvious that no ordinary person would even bother to assert it. But if this were a good reason for condemning a philosophical discussion about it as trivial, then much of logic would share this fate, since the fundamental principles of inference likewise are so 'obvious' that ordinary mortals do not bother to state them explicitly. [4]
}

Here Pap tries to refute the suggestion that the triviality of an example is a reason for regarding philosophical discussion of that example as trivial. He submits that the former is not a reason for the latter, because, if it were, then it would also be a reason for regarding discussions of the fundamental principles of logic as trivial. He seems to be saying that the argument in the second case is the same as the argument in the first, that it is implausible to see this as a good argument in the second case, and that it is therefore equally implausible to see it as a good argument in the first. What is in question is not whether 'the example is trivial' entails 'philosophical discussion of the example is trivial,' but rather whether the former is a reason to believe the latter.

b. Here the philosopher Heidelberger criticizes an argument used by Norman Podhoretz. Podhoretz, in the course of an extended discussion of the likely harmfulness of marijuana, wrote:

Second, marijuana can and does lead to heroin in a substantial number of cases, the number being roughly equal to the number of heroin addicts among us, since there are very few addicts to be found who did not begin by smoking marijuana, often at a relatively early age. [5]

\section{Heidelberger objected to Podhoretz' argument as follows:}

But of course it is equally true that there are very few heroin addicts to be found - not to mention users of the hallucinogens-who did not begin by drinking water, often at a relatively early age. What this means is that water can and does lead to heroin in a substantial number of cases-the number being roughly equal to the number of heroin addicts among us-and what this means is that water is a dangerous drug.[6]

In "Fallacies and the Evaluation of Reasoning," Maurice Finocchiaro discusses this dispute in more detail than I shall here and seems to follow 
Judith Thomson in holding that the logical analogy technique used by Heidelberger presupposes a deductivist interpretation of Podhoretz' argument. Precisely how Podhoretz' comments are best read I shall not venture to say. It seems to me, however, that the short argument quoted here can be taken as:

(1) There are very few heroin addicts to be found who did not begin by smoking marijuana.

So,

(2) Marijuana can and does lead to heroin use in a substantial number of cases.

and

(3) The number of cases in which marijuana use leads to heroin use is roughly equal to the number of heroin addicts there are.

(Implied)

therefore,

[4] Marijuana is a dangerous drug.

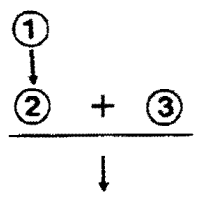

(4)

Heidelberger's argument would then be as above but with 'water' substituted for 'marijuana.' The parallel argument he constructs is totally implausible and makes the original one look ridiculous. Now my point about this whole discussion is a limited one. I do not think that Heidelberger's technique presupposes taking the above argument as deductive. The move from (1) to (2) seems to me to be broadly inductive - the causal claim of (2) being either an extrapolation from, or proposed explanation of, the correlation cited in (1). I believe that Heidelberger's parallel case casts doubt on this line of inference even when it is thus construed. This seems to me to be a tenable position because there are general considerations at issue in inferring causal conclusion from correlational premises, just as there are general issues at stake in deductive arguments.

I suggest, then, that the technique of logical analogy depends on, and reveals, the general connection on which an argument depends. It does not, in the narrow sense of "formal," depend on a formal connection. Nor does it depend on a deductive connection-whether this be achieved or only intended.

\section{Notes}

[1] Penelope Leach, Who Cares? (Middlesex, England: Penguin Books, 1979), p. 82.

[2] Stanley Cavell, The Claim of Reason (New York: Oxford University Press, 1979), p. 270. Cavell uses this type of argument very frequently. Additional examples may be found on pages 278-9 and 304-5 and, indeed, throughout the book.

[3] Of course philosophers have no non-arbitrary way of limiting this list of logical words. The "'and so on" clause is very important. It is sometimes said that logical words are syncategorematic. However, in the light of developing epistemic and deontic logics, some would accept "know", "believe", and "ought" as logical words. These are no more syncategorematic than "an" or "good" or "aceptable" or "produces", which are not commonly regarded as logical words. Compare discussions in Arthur Pap, Semantics and Necessary Truth (New Haven and London: Yale University Press, 1958), pp. 130-143 and 157 and Susan Haack, Philosophy of Logics (Cambridge: Cambridge University Press, 1978), pp. 22-27.

[4] Pap, Semantics and Necessary Truth, p. 153. 
[5] Quoted in Maurice Finocchiaro, "Fallacies and the Evaluation of reasoning," American Philosophical Quarterly, Vol. 18, \#1, pp. 13-22. p 21.

[6] ibid.
Dr. Trudy Govier, 3207 Canmore Road, N.W., Calgary, Alberta T2M $4 J 8$. 